\title{
Architectural Spaces as Living Organisms: Reflecting Inhabitants' Psychology and Behavior in the Space Through Embedded Responsiveness and Intelligence
}

\author{
MONA GHANDI \\ Washington State University
}

\begin{abstract}
Advances in computational algorithmic design, data sensing, information technology, material science, and fabrication technology have enabled architects to create adaptive spaces. Despite the high level of efficiency and sustainability that have been introduced through adaptive thinking, users' physiological and neurological needs is an area yet to be further explored. The objective of this paper is to offer an approach to democratize design and rethink the conventional rigid, solid architectural spaces in favor of structures that respond to users' deeper levels of engagement such as thoughts and feelings. It contributes to the design of future spaces that will be considered living organisms as they learn users' behavior and respond to their deeper needs and desires in real-time. This interdisciplinary research aims to create spaces whose capacity to resonate with immaterial aspects of the human, such as cognitive synapses and feeling, make them particularly compassionate. It explores the future of our intelligent environment through embedded responsiveness, drawing upon connections between architecture, data science, and other related technological advances. "Smart Architecture" lies at the center of its inquiry, examining the impact of robotized self-adjusting structures, relying on programmable materials and sensory network to allow architectural spaces to change and respond to real-time data in a fluid manner. Of its many consequences, the project has significant ethical implications, specifically as related to the medical field, tackling problems such as individuals with disabilities, neuromuscular diseases, motor system disorders, PTSD, and autism, ultimately empowering individuals with these deficiencies to regain control over their environments.
\end{abstract}

\section{INTRODUCTION}

\subsection{BACKGROUND AND PRECEDENT}

In an ever-changing social world, adaptive systems in architecture address contemporary needs of cities and citizens by responding to the changes in the user preference and environment. The increasing practicality of the Internet of Things (IoT), artificial intelligence (AI), active mechanical components, algorithmic design capabilities, material innovation, and robotic fabrication have also had a major impact in creating adaptive spaces that can change and respond to the users' need and environmental consideration in a fluid manner.
Merging information systems with wireless sensor networks, transceivers, and physical actuators, the Internet of Things (IoT) allows the physical devices to respond to one another and to human participants, either directly or remotely. Our physical environment is on the verge of becoming an extension of the Internet (Atzori et al. 2010; Gubbi et al. 2013; Vermesan \& Friess 2014). Integrating with homes, industrial workplaces, hospitals, energy grids, and traffic management systems (Bellavista et al. 2013), loT has much to offer humanity, including great improvements in efficiency in the areas of energy consumption, traffic congestion, industrial monitoring, waste management, and city infrastructure. Such applications of the loT in civic infrastructure have been referred to as the rise of "smart cities" (Schaffers et al. 2011; Zanella et al. 2014).

Adaptive thinking has transformed conventional rigid architectural spaces to an unprecedented level of dynamism by addressing the need for flexibility and reconfiguration while enhancing building performance, optimizing resource use, and increasing human interaction. Scholars have analyzed and categorized adaptive systems in a variety of ways. Fox and Yeh (2000), for example, classified kinetic architecture into "deployable," "dynamic," or "embedded" systems. Ramzy and Fayed (2011) proposed an alternative categorization based on the extent of kineticism, the adaptive system's configuration, and the specific mechanical techniques used. (Ramzy \& Fayed 2011; Arenas \& Falcón 2013). The Internet of Things and related computation technology merge seamlessly with the goals of adaptive architectural systems, providing tools to enhance the environmental quality of buildings and promote more flexible, human-centered designs.

\subsection{PROBLEM AND GAP IN THE LITERATURE REVIEW}

Current adaptive systems in architecture are used to create hybrid spaces that are dynamic and responsive to internal and external changes. Such spaces are highly efficient and sustainable, and possess complex structures; however, more awareness is needed on adaptivity and responsiveness to users' physiological and neurological needs.

Future architectural design requires solutions to integrate people, structures, and sensing technologies to arrive at a successful Human-computer interaction. (Beilharz 2005) Current examples of adaptive design range from those that respond 
to environmental forces (Hydramax, Future Cities Lab) and climate (HygroScope, Menges and Reichert) to the ones that respond to human engagement, motion, and sound (Petting Zoo, Minimaforms, Lightswarm, Future Cities Lab). While projects such as Murmur wall (reveals data/text via search engines and social media, Future Cities Lab) and Radiant Soil (suspended filter layers contain a near-living carbon-capture metabolism, Philip Beesley) tend to more invisible and intangible factors, the relationship between our psychology, thoughts, and built environment is rather less explored.

Kinetic approaches have mostly been used in façade reconfiguration for energy efficiency purposes, or in roofs over stadiums and exhibition spaces, opening and closing to fend weather, and in general toward environmental considerations but not toward specific users' needs.

Despite the high level of efficiency and sustainability introduced through adaptive thinking, difficulties stemming from physiological and neurological limitations is an area that is yet to be explored. This research aims to fill that gap, focusing on developing tangible reciprocities between users on the one hand and physical objects on the other, ultimately creating a world that is empathic and empowering.

\subsection{OBJECTIVE AND SIGNIFICANCE}

The objective of this research is to rethink the conventional rigid, solid architectural spaces in favor of structures that respond to our psychological needs. The significance of this project is the focus on the link between the mind and the space it inhabits to produce user-oriented built-environments.

This research aims at creating built environments treated less as features independent of the human condition but as entities in constant communication with it. Here walls windows and doors conspire with smart technologies to make space more responsive and compassionate. What used to be a static environment composed to appeal merely to the eye is here mobilized to solve invisible and yet real issues. Medical deficiencies in the self, such as autism and PTSD, are seen to lie and be the concern of architecture. Might our physical constructs move and change to address problems of cognition or mood? The question is difficult but this study takes it as its ethical challenge to see how it can be addressed.

Architecture can "feel" the environment and generate an empathetic relationship between users and their environment through a unification of materials, form, structure, and interactive systems of control. It fosters a process in which synapses in the brain trigger a response in buildings that could ultimately modulate emotion and heighten the level of intimacy between mind, body, and environment to dissolve their boundaries and make a single entity.
This paper's contribution is toward the design of future spaces conceived as living organisms, creating a cyclical loop between learning human behavior and a real-time response commensurate with it, using embedded responsiveness and material intelligence.

This interdisciplinary research draws upon connections between architecture, data science, neuroscience, material science, robotic technology, computer science, and artificial intelligence to create companionate spaces. Such spaces are intended to enable individuals with disabilities, motor systems disorders, autism, PTSD, neuromuscular diseases, and elderlies to live in adaptive spaces controlled by their minds and senses.

Specifically, by looking at the problems of PTSD (Posttraumatic Stress Disorder) and Autism (experiencing social-interaction difficulties and communication challenges), where people experience deficiency in their ability to express desire, needs, and feelings, this research can serve as an augmentation tool to compensate for those lacks, shifting the role of architecture from a discipline strictly concerned with space and aesthetics to one whose operation are first and foremost communicative and interactive. Which means that despite the fact the project demands technological skills it is fundamentally ethical in reach, seeking to siphon technology to level the ground in a world that is all too often unequal.

To achieve the goal of making our thoughts materially tactile, following objectives are put forward:

(1) Algorithmic design of a structure based on user data and material behavior.

(2) A data collection and actuation system comprised of sensors, actuators, and mobile applications.

(3) Fabrication and activation of a self-adjusting structure/ wall using robotic fabrication methods, incorporating active shapes, flexible materials, soft robotics, smart and programmable materials, and pneumatic systems. (Figure 1)

\section{METHODOLOGY}

To achieve the goal of the project, this research requires a multi-facet approach. Part of it is engaged in a sensory network of collecting data and understanding human condition (Emotional computing, Affective computing, Expression recognition) while other parts involve structures, actuation systems, and materials that can respond in kind.

\subsection{COLLECTING DATA AND UNDERSTANDING HUMAN CONDITION}

For the first part, collecting data and understanding human condition, the approach used in this project is to equip users and the environment with smart embedded devices that can 

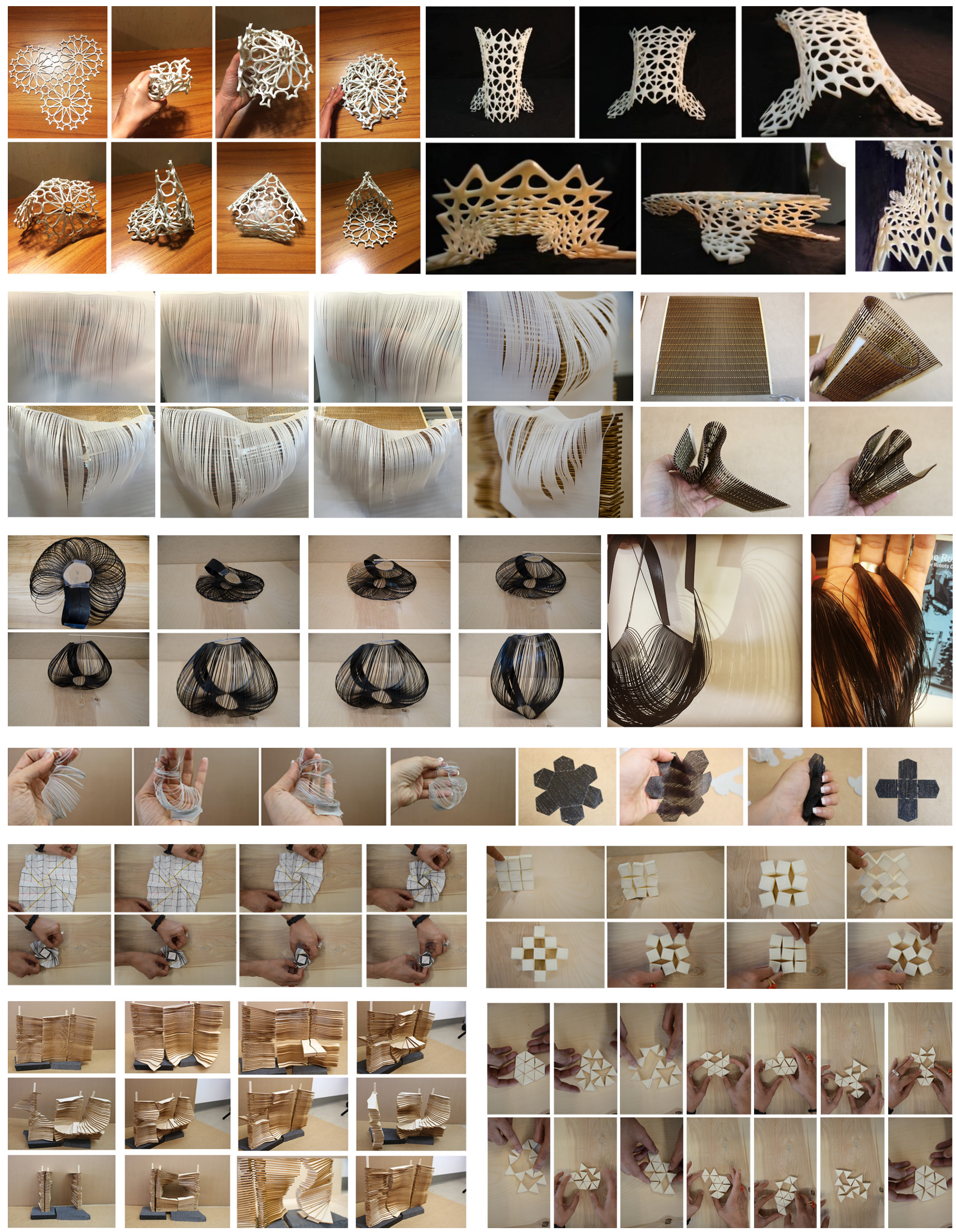

Figure 1. Explorations of inherit flexible behaviour of materials, active shapes, transformable structures, robotic 3D printing of flexible materials for intended shape changing 
collect users' real-time behavioral data, mental and physical, within the realm of loT.

As mentioned before this research has two types of applications:

\subsubsection{COLLECTING PHYSICAL DATA}

The first application is to help people with disabilities and elderlies to provide them more control over their environment. Here gesture and motion detectors, eye trackers, voice recognition, and smart devices are used to collect data and transfer it to an adaptive wall/structure that can reconfigure its shape to the new states that can serve those people better. Users will have the opportunity to create windows based on their desire, change the size of the opening, adjust the view, control the light, and natural ventilations, or change the wall shape according to their programmatic needs. In this part, users can communicate with their built environment through voice, vision, touch, and movement.

\subsubsection{COLLECTING EMOTIONAL DATA}

The second application is to help people with PTSD and Autism who face deficiency in their ability to express their desire, needs, and feelings. This research can contribute to nurturing the senses and desires, that people have difficulty expressing, and reflecting them in the built environment. Here the builtenvironment is considered as a medium of interaction. To achieve expression, mood, and emotional recognition, brain activity trackers (EEG Headset), face recognition programs, voice detection devices, and wearable sensors are used to collect the biological data and recognize the moods and feelings of the users based on those data. These devices map users' emotion by gathering any relevant emotional data such as heart rate, electrical changes in the skin, blood pressure, body temperature, etc. These collected data will be transferred to architectural components causing them to change and modify. These changes in the structure/wall could be used as a communicative tool or as a means of helping the user to change, heal, or improve existing mood.

\subsection{PROCESS OF STRUCTURES, ACTUATION SYSTEMS, AND MATERIALS EXPLORATION}

To begin, data is registered by sensors and processed by algorithmic and generative design. Afterward, decisions are made by programmed controllers and actions are performed through actuators, programmable materials, and soft robotics. This embedded responsiveness is represented by a tectonic shift in the skin of the wall (materials), its cells (geometry) and its bone (structure).

The structure implements kinetic components and surfaces to perform certain reconfiguration such as seamlessly opening or closing upon receiving commands. In doing so, Rhino 3D modeling and its graphical algorithm editor (Grasshopper), Ghowl, and Python are used to incorporate data as input. The algorithmic logic is used to perform predefined operations, calculate the results, perform simulations, evaluate the design strategies, and subsequently to generate an optimized parametric design output.

Sensors are embedded in the structure itself or on devices that are voice, touch, gesture, or vision-activated, such as mobile apps, eye trackers, speech recognition software, motion, and brain activity tracking sensors, such as EEG headsets. As a result, real-time data is collected from users' cognitive and biological state, sent to embedded microcontrollers, and forwarded to the actuators.

This project uses soft robotics and pneumatic system, programmable materials, SMA (Shape Memory Alloy), and 'hard' components for actuation and structural transformation. Materials are studied and exposed to elements such as heat, light, and air pressure to activate kinetic components and achieve maximal geometric transformation. Finally, wall sections, and adaptive reconfigurable frame structures that host components are spatially extruded through non-layer based robotic 3D printing processes. (Figure 2)

\section{RESULT}

By focusing on the concept of Smart Architecture that utilizes robotized and self-adjusting structures, we are trying to implement(i) mental and physical sensing elements embedded in the structure or provided to the user through mobile and static devices; (ii) actuators that perform based on the data received from the sensors and sent via applications on smart mobile devices; and (iii) partially and fully reconfigurable structures.

The transformable wall to be prototyped, as an example, reconfigures itself based on programmatic, environmental, and mental needs of its users through following preparations.

\subsection{WALL DESIGN}

Two transformable structures and wall prototypes were made for this project. One is made from two vertical and horizontal panels carrying 3D printed flexible materials that move with servos on rails and change their positions based on the location and size of the window. This wall will update the location and size of the window with data received from vision, gesture, voice, and touch. The use of microcontrollers like Arduinos, firefly shields, and actuators like servos made this adaptivity happen. (Figure 3)

The other wall is made of triangular modules that can change their positions and create a new configuration based on the new data. This wall reconfigures its shapes based on different emotional data collected through eye tracking, voice detection, face recognition, EEG headset, biological data with wearable devices. Different actuators like SMA (Shape memory alloy), Servos, soft robotic experimented for this prototype. (Figure 4) 

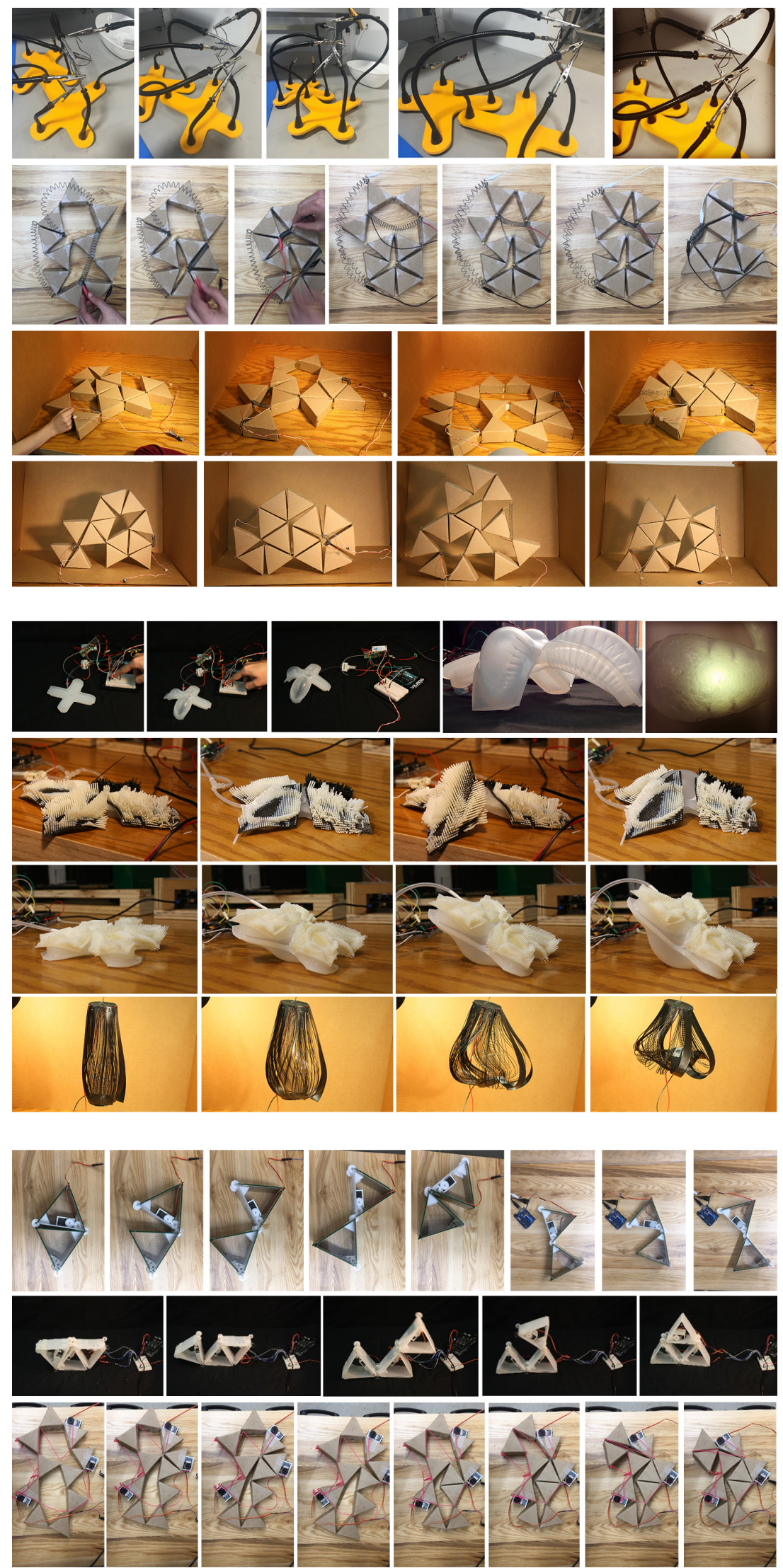

Figure 2. soft robotics and pneumatic system, programmable materials, SMA, and servo studies for actuation and structural transformation. 

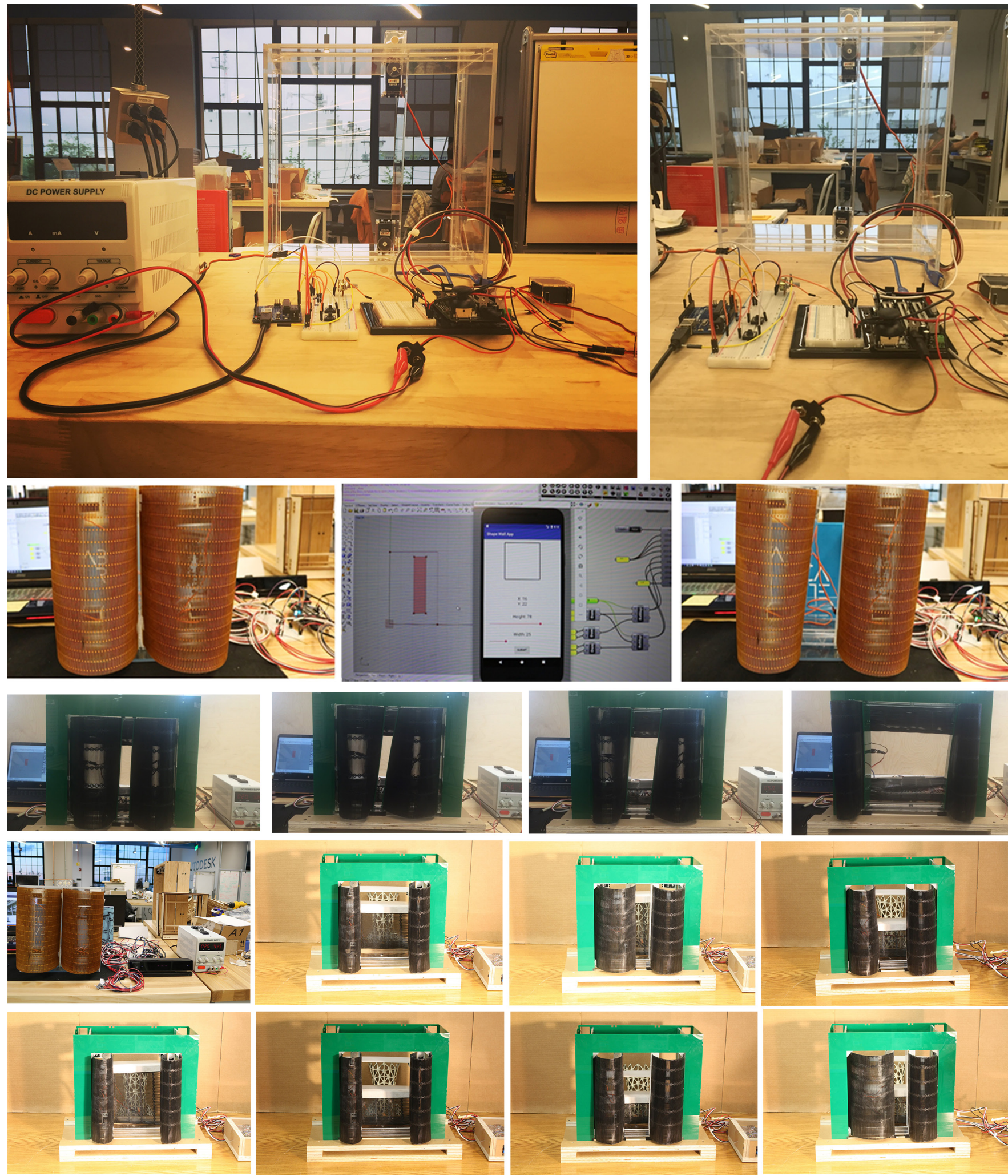

Figure 3. Wall prototype with adjustable window that has automatic responses to emotional and mental data collected from the user. Users can change the size and location of window based on their needs 


\subsection{SERVER DESIGN}

Using a function in a Java library called "Stream", data is sent to the server. To accomplish different forms of input to modify the wall, our design centers around having input from all peripherals sent to the server which updates to separate sets of data. The 'master' set of data is what Grasshopper uses to generate the virtual wall. The 'preview set' is where changes are stored as an XML file before the 'master' dataset is updated. Through a URL which uses javascript and html5 a visual representation of the preview data is displayed. Accessed by most web browsers, users can see the changes on the wall before submitting it to the 'master' set and ultimately having those changes reflected in grasshopper.

\subsection{FRONT-END AND GRASSHOPPER BACK-END}

In the front-end, data will be collected through Android App, Alexa Skill, Kinect App, Tobii Eye Tracking App, Affectiva face recognition web, wearable wristband, and Emotive EEG Headset to interact with the wall. Moreover, we are exploring the ways that let grasshopper receive information sent from the front-end app and convert the information to data that create the window or change the configuration of the wall. The Rhino base software would not accept data sent from the server that holds the sensors' data. To resolve this, the extensions Grasshopper, Ghowl and Firefly extensions are used to allow Rhino to accept data points via code from the server. The back-end uses Ghowl to ping the server every 5 seconds and request for the data in the XML format. Then, the information is verified.

\subsection{TOUCH}

An Android App created using Java and Android Studio allows users to touch a square that represents the wall and place a window on the wall. The window can be moved based on where the user taps in the square. Users can move sliders that represent the width and the height of the wall for further adjustment or they can choose their moods and feeling to apply changes in the wall configurations. Finally, users can submit the wall to the preview and to the final version of the wall. The application works by sending HTTP requests containing the new coordinates for the window or new wall configuration to the server. The app supports actions such as window placement, window dimension change, wall configuration and submission to the preview and to Grasshopper.

\subsection{VOICE}

Amazon Alexa voice service is used to interpret voice commands. Our Alexa Skill works by sending speech converted to text to an application created on AWS Lambda using Node.js. The app first retrieves the current coordinates of the window via HTTP get request, and based on the received speech text, the app modifies the coordinates and sends them back to the server. Certain "utterances", or spoken phrases are used with Alexa as "intents" or actions executed by Alexa. The intents have utterance mapped to them. For instance, "Move it [up/ down/left/right]" is mapped to the "move" intent. The app in its current state supports utterances such as 'Alexa, Ask Shape Wall', 'move it', 'make it', 'Make a window', and 'submit', which submits the wall to grasshopper from the preview. Alexa also uses some utterances about the feeling and moods of the users like "I am happy, sad, scared, depress, angry, etc." and executes some configurations assigned to those moods.

\subsection{GESTURE}

Kinect sensor is used to track human movement through a program that attaches a skeleton to the user. It logs gestures by recognizing 25 joints across the body. The program recognizes specific shapes and movements such as a closed fist, open palm, and the lasso, and the shapes are combined with basic swiping movements to create gesture commands. Moving each hand with one of the shapes moves the box or increases and decreases the dimensions of the box according to how they are programmed. After satisfactory changes, users submit the image and after 5 seconds of inactivity, data will be sent to the server.

\subsection{VISION}

Data is collected with Tobii eye tracker to track users' eyeball movement such as gaze point location and blinking behavior to control our models. The Tobii eye SDK does provide blink detection so that we implement the blink event with the following logic:

- Whenever the eye position is out of scope (eye closed), record the current system time as t0

- Whenever the eye position is in the scope (eye opened), record the current system time as $\mathrm{t} 1$

- If $\mathrm{t} 1-\mathrm{t} 0$ > $=300 \mathrm{~ms}$ (i.e. the previous eye closed time lasts at least $300 \mathrm{~ms}$, a single blink event detected), record the current system time as e 0

- If more than 400 ms past after e0 and no new eye blink event detected, activate the function that sent according to configuration code to server, otherwise activate the function for double eye blink

Data retrieved from the Tobii eye tracker will be translated to various motions to change the location and size of the window, to open and illuminate the selected spots, or to change the configuration of the wall.

\subsection{FACE RECOGNITION}

We used Affectiva face recognition for mapping the moods and feelings of the users through facial expression. The Affectiva SDK is a pure software solution which can run on any computer with a camera built in. The Affectiva SDK uses the built-in camera to detect users' expression (such as happy, anger, etc.) and bundles those emotion state in a JSON object. We write JavaScript code to extract the JSON object from the Affectiva SDK and send it back to our server. On the server 


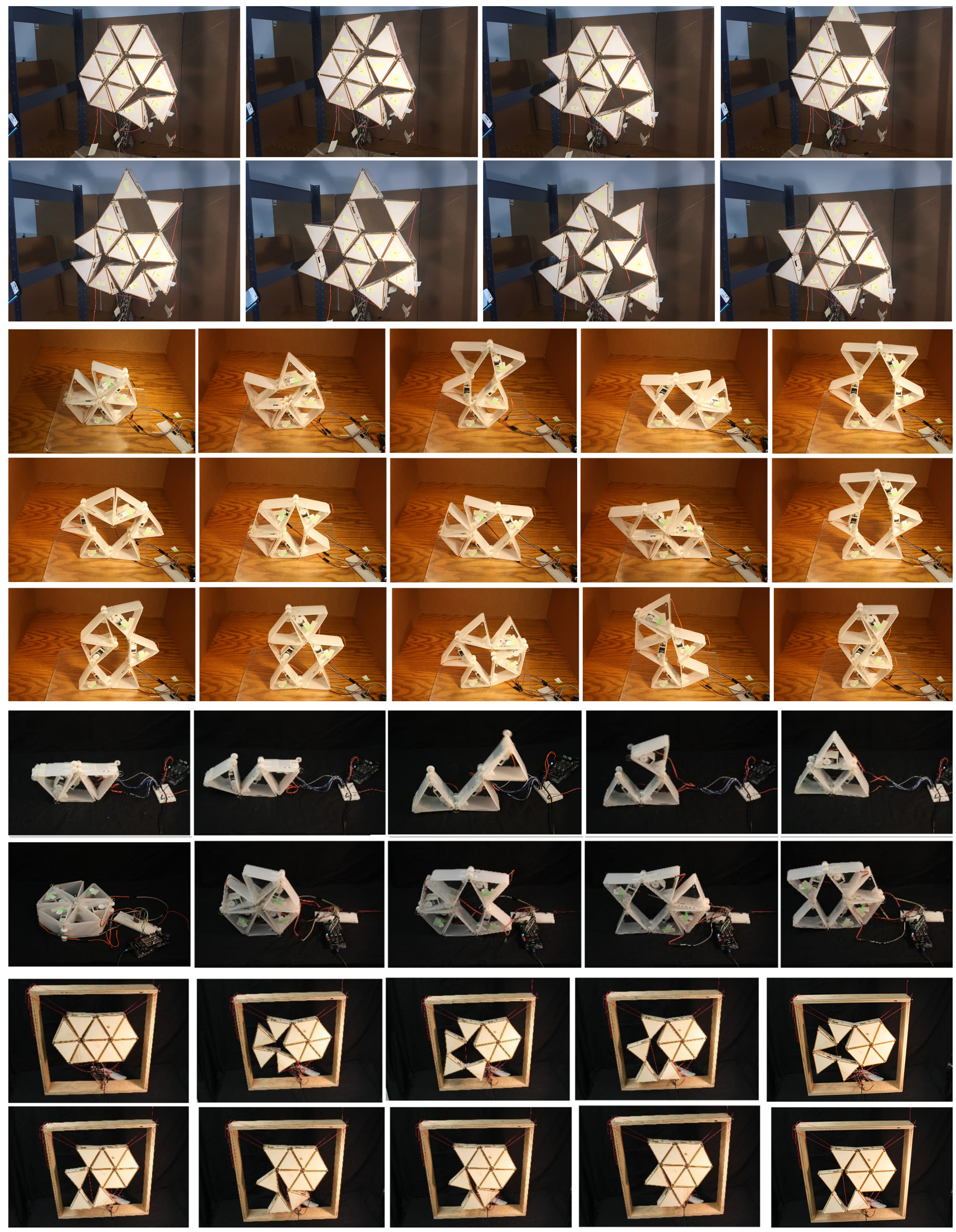

Figure 4. Adaptive Wall prototype with adjustable triangular modules that changes its configuration based on emotional and mental data from the user 

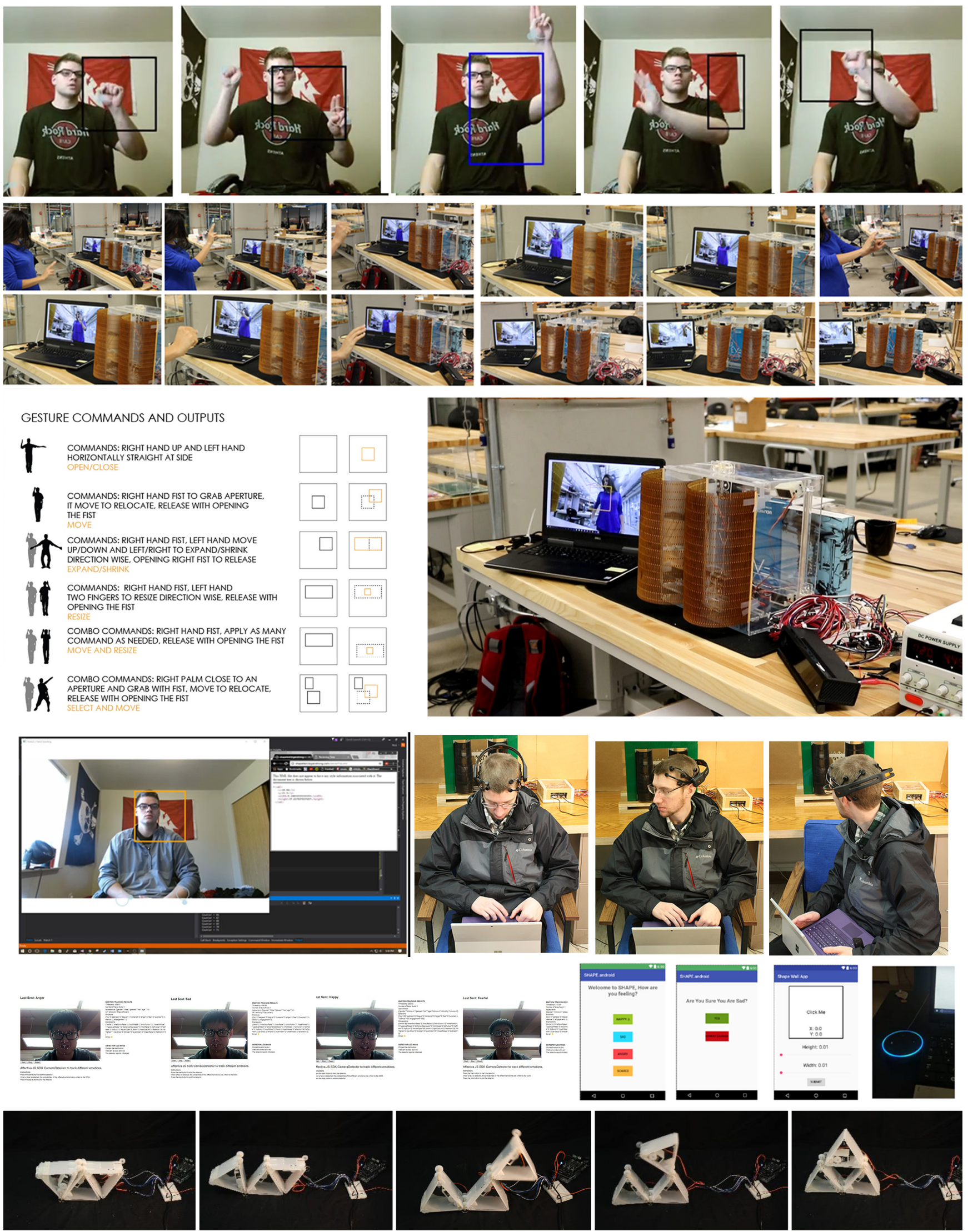

Figure 5. Gesture, facial expression, eye tracker, voice, mental, and App commands and both adaptive walls responses 
side, the received JSON object will be parsed into correlated configuration codes to update the wall to the new status. (Figure 5)

\subsection{EMOTIVE WEARABLE DEVICES}

Development for the mood and feeling tracking using the wearable devices is in the very early stages. Emotive wearable devices are cable of collecting any relative biological data (such as heart rate, electrical changes in the skin, blood pressure, body temperature, breathe rate, Oximeter, cardiac electrical potential waveforms, etc.) to map the mood and feeling of the users. For now, we are capable of mapping 3 feelings (Happy, excited, scared) to change the configuration of the wall. Developing methods for more data interpretation to change the configuration of the wall is in the process.

quis gravida dolor, vitae commodo orci. In dictum sem ut aliquam semper. Cras imperdiet et diam in luctus. Nullam porta lectus non vehicula scelerisque. Donec efficitur, elit hendrerit sagittis sagittis, est arcu dignissim lectus, eget bibendum enim arcu sodales nunc. Vestibulum lacinia, tellus a vulputate pharetra, lacus est tincidunt massa, eu maximus ipsum nulla nec diam. Proin ac hendrerit turpis. Sed ac molestie lacus.

\section{FUTURE DEVELOPMENT}

This is an ongoing research. The first step of collecting data (vision, voice, touch, and gesture, face, biological data) via sensors, trackers, and smart devices, storing them in a server, transferring them (to Grasshopper), previewing the changes, and sending them to microcontrollers and actuators, projecting the changes in the prototype is completed.

In addition to reevaluation and improvement of what has been done, the further development will focus on improvement of the wall design more towards soft robotics and pneumatic system. The other development of this research will focus on cognitive and sensory network infrastructure. By focusing on the users' psychological, behavioral, and neurological data collected through sensors that can detect brain neural activities and responses, this research will seek for automated responses of the architectural entities through the synaptic network effects.

The advanced goal will be exploring the ways in which synapses in the brain can trigger an automatic response in buildings that could ultimately modulate emotion. This will help people with disabilities to control their environment with their mind.

Next step would be how we can create smart spaces that can not only interact with the users' need and respond to their deeper needs and desires in real-time, but they can also learn user's behavior and autopilot the required changes in the environment based on the users' behavioral patterns. Our future development can take advantage of machine learning techniques to offer such spaces.

\section{CONCLUSION}

By showcasing the application of data-driven design strategies within human-computer interaction in adaptive spaces, this paper presents an example of innovations in architecture made possible by our current technological environment and through an exciting collaboration with computer science, neuroscience, material science, robotics, and other related fields. This paper presents an alternative method by which to transcend the limitations of our physical environment. In fact, it aims to empower the users by understanding and accommodating their needs. It helps to democratize our design process and let the users have a greater role in shaping their built environment.

Existing limitations in our built environment could be challenged by pursuing more user engagement to blur the line between the structure as an autonomous entity and the inhabitants of the space. This project aims to rethink technological ethics in architecture in pursuit of a more just built environment and achieve seamless levels of structural adaptivity based on deeper levels of user engagement. Our approach is a framework for integrating adaptive systems and data-driven approaches into a design and transform the built environment into a living organism that is networked, intelligent, sympathetic, sensitive, adaptive, and yet under the comprehensive control of the user. Drawing upon loT, robotic fabrication, material innovation, and behavioral sciences, mind and body-controlled spaces can become spaces of the future.

The outcome of this ongoing research is a platform for designers to study and create amid the new possibilities of architecture in the current technological moment. Rather than suggesting an ultimate solution for kinetic architecture needs, this research is a stepping-stone to endless design possibilities.

\section{ACKNOWLEDGMENT:}

"This research project has been supported by AUTODESK BUILD GRANT, under the IAAC/AutoDesk BUILD Space Residencies"

Special thanks to Hamidreza Esmaeillou and Yang Zhang, who assisted me with coding and programming, research, fabricating models, documenting, and drawings. Thanks to Patrick Bowling, Aaron Kwan, Andrew Lewis, Jonah Simon, and Nick Strazis for their help with coding and programming. Thank you to Behrooz Shirazi for all of his support on the project. 


\section{REFERENCES}

1 Arenas, Ubaldo, and José Manuel Falcón. 2013. “Adaptable Communication Protocols for Robotic Building Systems". In ACADIA 13: Adaptive Architecture [Proceedings of the 33rd Annual Conference of the Association for Computer Aided Design in Architecture (ACADIA), Cambridge 24-26 October, 2013): 237-243.

2 Atzori, Luigi, Antonio lera, and Giacomo Morabito. 2010. "The Internet of Things: A survey." Computer Networks 54 (15):2787-2805. doi: 10.1016/j. comnet.2010.05.010.

3 Beesley, Philip. 2009. “Hylozoic Soil.” Leonardo 42 (4):360-361. doi: 10.1162/ leon.2009.42.4.360.

4 Beilharz, Kirsty. 2005. "Responsive Sensate Environments: Past and Future Directions." In Computer Aided Architectural Design Futures 2005: Proceedings of the 11th International CAAD Futures Conference held at the Vienna University of Technology, Vienna, Austria, on June 20-22, 2005, edited by Bob Martens and Andre Brown, 361-370. Dordrecht: Springer Netherlands.

5 Bellavista, Paolo, Giuseppe Cardone, Antonio Corradi, and Luca Foschini. 2013. "Convergence of MANET and WSN in loT Urban Scenarios." Sensors Journal, IEEE 13 (10):3558-3567. doi: 10.1109/JSEN.2013.2272099.

6 Besenecker, Ute Christa, and Ted Krueger. 2015. Luminous Color in Architecture: Exploring Methodologies for Design-Relevant Research. The ARCC Journal 12(1): 35-46. http://dx.doi.org/10.17831/enq:arcc.v12i1.392

7 Fox, Michael A., and Bryant P. Yeh. 2000. "Intelligent Kinetic Systems in Architecture." In Managing Interactions in Smart Environments: 1st International 2. Workshop on Managing Interactions in Smart Environments (MANSE'99), Dublin, December 1999, edited by Paddy Nixon, Gerard Lacey and Simon Dobson, 91-103. London: Springer London.

8 Future Cities Lab, San Francisco. 2016. “Murmur Wall." accessed May 20, 2017. http://www.future-cities-lab.net/murmurwall/.

9 Future Cities Lab, San Francisco, Jason Kelly Johnson, Nataly Gattegno, and Ripon DeLeon. 2016. "Lightswarm." accessed May 20, 2017. http://www.futurecities-lab.net/lightswarm/.

10 Gattegno, Nataly, and Jason Kelly Johnson. 2015. "Sentient Futures: The Hydra Trilogy by Future Cities Lab." Architectural Design 85 (4):74-79. doi: 10.1002/ ad.1929.

11 Gubbi, Jayavardhana, Rajkumar Buyya, Slaven Marusic, and Marimuthu Palaniswami. 2013. "Internet of Things (IoT): A vision, architectural elements, and future directions." Future Generation Computer Systems 29 (7):1645-1660. doi: 10.1016/j.future.2013.01.010.

12 Menges, Achim, and Steffen Reichert. 2015. "Performative Wood: Physically Programming the Responsive Architecture of the HygroScope and HygroSkin Projects." Architectural Design 85 (5):66-73. doi: 10.1002/ad.1956.

13 Ramzy, Nelly, and Hatem Fayed. 2011. "Kinetic systems in architecture: New approach for environmental control systems and context-sensitive buildings." Sustainable Cities and Society 1 (3):170-177. doi: 10.1016/j.scs.2011.07.004.

14 Schaffers, Hans, Nicos Komninos, Marc Pallot, Brigitte Trousse, Michael Nilsson, and Alvaro Oliveira. 2011. "Smart Cities and the Future Internet: Towards Cooperation Frameworks for Open Innovation." In The Future Internet: Future Internet Assembly 2011: Achievements and Technological Promises, edited by John Domingue, Alex Galis, Anastasius Gavras, Theodore Zahariadis, Dave Lambert, Frances Cleary, Petros Daras, Srdjan Krco, Henning Müller, Man-Sze Li, Hans Schaffers, Volkmar Lotz, Federico Alvarez, Burkhard Stiller, Stamatis Karnouskos, Susanna Avessta and Michael Nilsson, 431-446. Berlin, Heidelberg: Springer Berlin Heidelberg.

15 Spyropoulos, Theodore, and Stephen Spyropoulos. "Minimaform Projects: Petting Zoo." http://minimaforms. com/\#item=petting-zoo-barbican-centre-london.

16 Vermesan, Ovidiu, and Peter Friess, eds. 2014. Internet of things applications - from research and innovation to market deployment. Gistrup, Denmark : River Publishers. http://www.internet-of-things-research.eu/pdf/IERC_Cluster Book_2014_Ch.3_SRIA_WEB.pdf

17 Zanella, Andrea, Nicola Bui, Angelo Castellani, Lorenzo Vangelista, and Michele Zorzi. 2014. "Internet of Things for Smart Cities." Internet of Things Journal, IEEE 1 (1):22-32. doi: 10.1109/JIOT.2014.2306328 